\title{
Fast error-safe MOID computation involving hyperbolic orbits
}

\author{
Roman V. Baluev \\ Saint Petersburg State University, Faculty of Mathematics and Mechanics, Universitetskij pr. 28, Petrodvorets, Saint Petersburg \\ 198504, Russia
}

Central Astronomical Observatory at Pulkovo of the Russian Academy of Sciences, Pulkovskoje sh. 65/1, Saint Petersburg 196140, Russia

\begin{abstract}
We extend our previous algorithm computing the minimum orbital intersection distance (MOID) to include hyperbolic orbits, and mixed combinations ellipse-hyperbola. The MOID is computed by finding all stationary points of the distance function, equivalent to finding all the roots of an algebraic polynomial equation of 16th degree. The updated algorithm carries about numerical errors as well, and benchmarks confirmed its numeric reliability together with high computing performance.
\end{abstract}

Keywords: close encounters, NEOs, catalogs, computational methods

\section{Introduction}

The MOID parameter, or the minimum distance between points on two Keplerian orbits, is an important practical tool measuring the closeness of two Keplerian trajectories in the $\mathbb{R}^{3}$ space. This parameter is frequently used in studies of Potentially Hazardous Objects (PHOs) and Near-Earth Objects (NEOs), see e.g. (Sitarski, 1968; Dybczyński et al., 1986). Also, the MOID and MOID-like quantities can be used to estimate possible visibility condition of one object from another (Gronchi and Niederman, 2020).

The MOID is a minimum of some distance or distance-like function $\rho\left(u, u^{\prime}\right)$ that depends on two arguments, determining positions on two orbits. Multiple methods of finding the minima of $\rho\left(u, u^{\prime}\right)$ are available Kholshevnikov and Vassiliev (1999); Gronchi (2002); Baluyev and Kholshevnikov (2005); Gronchi (2005); Armellin et al. (2010); Hedo et al. (2018); Baluev and Mikryukov (2019), as well as methods allowing to put useful bounds on the MOID or related quantities (Mikryukov and Baluev, 2019, Gronchi and Niederman, 2020).

Email address: r.baluev@spbu.ru (Roman V. Baluev)
The fastest methods appear those in which both $u$ and $u^{\prime}$ are solved for rather than found by numeric optimization (Kholshevnikov and Vassiliev, 1999, Gronchi, 2002, 2005; Baluyev and Kholshevnikov, 2005; Baluev and Mikryukov, 2019). The task is analytically reduced to solving a nonlinear equation with respect to $u$ and then expressing $u^{\prime}$ also analytically. However, methods of this class are relatively vulnerable with respect to numeric uncertainties through loosing real roots in nearly degenerate cases. This effect appears because the equation for $u$ is quite complicated and has algebraic degree of 16 in the general case. It often have close (almost multiple) roots that are always difficult for numeric processing.

The recent work by Baluev and Mikryukov (2019) represents an efficient solution of this issue based on careful treatment of numeric errors appearing on the way. Basically, it represent a numeric implementation of the algebraic approach presented by Kholshevnikov and Vassiliev (1999), similar to the one presented by Gronchi (2002, 2005). However, this newer algorithm is only capable to process elliptic orbits. This appears because the arguments $u$ and $u^{\prime}$ have the meaning of eccentric anomaly that 
is not sensible on a hyperbolic orbit. This contrasts with (Gronchi, 2005) where this issue does not appear because they used true anomalies as free variables. However, that code revealed numeric unreliability on some orbit pairs (Hedo et al., 2018; Baluev and Mikryukov, 2019). Though the fraction of such cases is small, they often escape from being selfdiagnosed, making such code less prefered than alternatives that avoid failures. Simultaneously, the Gronchi code appears somewhat slower than the alternatives from that works.

In this work we have a goal to extend the numerically stable and fast code from (Baluev and Mikryukov, 2019), making it capable to process hyperbolic orbits as well. This relies on the work by Baluyev and Kholshevnikov (2005), where the analytic theory from (Kholshevnikov and Vassiliev, 1999) was extended to all types of Keplerian orbits in any combinations. The need of processing the hyperbolic orbits is highlighted by recent discoveries of new interstellar objects passing close to Sun. In particular, the cometary object 2I/Borisov has an orbital eccentricity above 3 , and its trajectory passes through the inner part of Solar system (Guzik et al. 2019). Moreover, early orbital solutions proposed that its could be a NEO. It was thus listed on IAU's Minor Planet Center's NEO Confirmation Page as gb00234. Therefore, one may consider the task of quick computation of all MOIDs between such an object and Main belt orbits with a goal to reveal asteroids that have a risk of collision or close approach. Small MOID does not guarantee such an event in itself, as the objects would need to also appear with proper orbital positions for a collision. But this approach might help to filter away those asteroids that do not have that chance at all.

The $\mathrm{C}++$ source code of our MOID library named DISTLINK is available for download at http://sourceforge.net/projects/distlink.

The structure of the paper is as follows. In Sect. 2, we discuss the mathematical framework for MOIDs of hyperbolic orbits. Sect. 3 describes the computing algorithm. Sect. 4 presents its performance tests.

\section{Mathematical setting}

Consider two confocal orbits: $O$ determined by the five geometric Keplerian elements $a, e, i, \Omega, \omega$, and $O^{\prime}$ determined analogously by the same variables with a stroke. We need to compute the minimum of the distance $\left|\boldsymbol{r}-\boldsymbol{r}^{\prime}\right|$ between two points lying on the corresponding orbits, and the orbital positions where this minimum is attained. In this work we consider elliptic $\mathcal{E}$ as well as hyperbolic $\mathcal{H}$ orbits, thus we have four possible combinations: $\mathcal{E} \mathcal{E}, \mathcal{E} \mathcal{H}, \mathcal{H} \mathcal{E}$, $\mathcal{H} \mathcal{H}$. Here we do not consider parabolic cases, as they appear degenerate both from the elliptic or hyperbolic point of view, resulting in reduced degree of the main polynomial. They require a special treatment therefore.

In the purely elliptic case the method by Kholshevnikov and Vassiliev (1999) reduced the problem to solving for the roots of a trigonometric polynomial $g(u)$ of minimum possible algebraic degree 16 (trigonometric degree 8 ). This polynomial can be expressed through the Keplerian elements of $O$ and $O^{\prime}$, and the associated formulae are omitted here for brevity.

For each root $g(u)$ we can determine the second position $u^{\prime}$ from explicit equations. In nondegenerate cases there is only a single value of $u^{\prime}$ that corresponds to a particular solution for $u$.

Finally, after both the orbital positions $u$ and $u^{\prime}$ were determined, the squared distance between these points is scaled as $\rho\left(u, u^{\prime}\right)=\left|\boldsymbol{r}-\boldsymbol{r}^{\prime}\right|^{2} /\left(2 a a^{\prime}\right)$. The adimentional function $\rho$ can be used to compare different solutions (roots of $g(u)$ ).

This central function $g$ can be rewritten in the standard trigonometric form:

$$
g(u)=\sum_{k=-N}^{N} c_{k} \mathrm{e}^{i k u},
$$

where $N=8$. By making the substitution $z=\mathrm{e}^{i u}$ or $w=\mathrm{e}^{-i u}$, we can transform it to:

$$
g(u)=\sum_{k=-N}^{N} c_{k} z^{k}=\mathcal{P}(z) w^{N}=Q(w) z^{N} .
$$

The task of finding roots of $g(u)$ becomes equivalent to solving the algebraic equation, $\mathcal{P}(z)=0$ or $Q(w)=$ 0 . All complex roots of $g(u)$ combine into conjugate 
pairs in terms of $u$, corresponding to the relationship $z \mapsto 1 / z^{*}$ in terms of $z$. Only $z$ with unit absolute value correspond to real $u$.

All coefficients $c_{k}$ can be formally expressed through Keplerian elements, but in practice this does not appear possible even using computer algebra. Still, a short explicit form for $c_{ \pm 8}$ is given in (Baluev and Mikryukov, 2019).

Cases when one of the orbits is hyperbolic or parabolic were considered in (Baluyev and Kholshevnikov, 2005). In the hyperbolic case, we should make a replacement

$$
u \mapsto i u, \quad \sqrt{1-e^{2}} \mapsto i \sqrt{e^{2}-1},
$$

where $i$ is the imaginary unit (not to be mixed with the inclination), and "new" $u$ attains the meaning of the hyperbolic analogue of eccentric anomaly.

The entire set of resulting formulae is given in (Baluyev and Kholshevnikov, 2005), and we do not replicate them all here. We only notice the following. In the $\mathcal{E H}$ case the polynomial $g$ preserves its usual trigonometric form (1), so the computing algorithm remains nearly unchanged. However, if the first orbit is hyperbolic, $g$ becomes

$$
g(u)=\sum_{k=-N}^{N} c_{k} \mathrm{e}^{-k u},
$$

and it is reduced to the algebraic form (2) with $z=$ $\mathrm{e}^{-u}$ or $w=\mathrm{e}^{u}$. Contrary to the elliptic case, all coefficients $c_{k}$ are real now, and $z$ should be real. Complex roots combine into conjugate pairs (in terms of $u$ as well as $z$ ).

In the elliptic case the number of real roots of $g(u)$ cannot be smaller than 4 , taking the multiplicity into account, because $\rho\left(u, u^{\prime}\right)$ is a continuous function defined on a torus (Kholshevnikov and Vassiliev, 1999). This appears because we should necessarily have at least one minimum and at least one maximum of $\rho$, and then the number of saddle points in a general (non-degenerate) case should be at least 2 following the Morse theory (Gronchi, 2005). The latter says that No. of maxima + No. of minima - No. of saddles = Euler-Poincaré characteristic (EPC) of the manifold (EPC $=0$ for torus). However, when one or both the orbits are hyperbolic, function $\rho$ is defined on a non-compact domain, either cylinder
$(\mathrm{EPC}=0)$ or plane $(\mathrm{EPC}=1)$. Also, we should treat each hyperbola branch separately, so the total number of critical points is doubled or quadrupled. In a mixed $(\mathcal{E H}$ and $\mathcal{H} \mathcal{E}$ ) case for each hyperbola branch we have at least one minimum, and hence at least one saddle point from the Morse theory, or 2 critical points per branch and 4 ones in total. In the $\mathcal{H} \mathcal{H}$ case we have at least 4 local minima (the number of possible branch combinations), and no informative limit on saddle points. Therefore, in all non-degenerate cases (no root multiplicity) we have at least 4 critical points in any orbital combination. Simultaneously, this number should be even, because complex roots always combine into pairs and the algebraic degree of $g$ is always even.

If at least $O$ is hyperbolic then there should be even number of roots per each hyperbola branch, and hence at least two roots per branch. This is because

$$
c_{8} c_{-8} \geq 0 \text {, }
$$

which is satisfied in any orbital combination. This property follows from the explicit expression for $c_{ \pm 8}$ given in (Baluev and Mikryukov, 2019). We consider now that $\mathcal{P}(z)$ is real-valued, and if $c_{8} c_{-8}>0$ then $\mathcal{P}(0)$ and $\mathcal{P}( \pm \infty)$ have the same sign. Hence, there are even number of positive and even number of negative roots, corresponding to either real or imaginary branch. In case when one (or both) $c_{ \pm 8}$ vanishes then there is one or more roots $z=0$ or $w=0$, corresponding to the $\mathcal{H} \mathcal{H}$-case with parallel asymptotes (Baluyev and Kholshevnikov, 2005). These zero roots can be arbitrarily attributed to either branch, so the number of roots per branch can always be treated even.

The upper limit on the number of real roots is uncertain even in the $\mathcal{E} \mathcal{E}$ case. It cannot exceed 16 , the algebraic degree of $g(u)$, but simulations never revealed more than 12 real roots (Kholshevnikov and Vassiliev, 1999; Baluev and Mikryukov, 2019). Sometimes we obtained 14-root and even 16-root occurrences using the standard DOUBLE precision, but with LONG DOUBLE arithmetic these cases appeared to have no more than 12 real roots. Moreover, in the testcase considered below we likely had none numerically reliable cases with 12 real roots. Considering hyperbolas, we detected a small fraction of quite reli- 
able 12-root occurrences, but when restricted to only $z \geq 0$ (the main branch of $O$ ), we had only 8 real roots at most in the $\mathcal{H E}$ case, and 10 at most in the $\mathcal{H} \mathcal{H}$ case.

Since the number of roots can be highly variable, it is a frequent case when there are close roots that cannot be easily resolved due to numeric errors. This effect was important in the $\mathcal{E} \mathcal{E}$ case, where it could lead to lost roots of $g(u)$, due to their misclassification as complex rather than real. This effect lead sometimes to overestimated distance because some critical points might appear lost. The solution was to track numeric errors of the roots and to select the real ones based on their estimated numeric uncertainty (so that some formally complex roots of $g(u)$ with small imaginary part could be tested together with purely real ones). In this work we expand this same approach to the hyperbolic orbits as well.

\section{Numerical algorithm}

The general computing sequence remains the same as in (Baluev and Mikryukov, 2019).

1. Compute the coefficients of $g(u)$ and their numeric uncertainties by means of the Discrete Fourier Transform (DFT).

2. Determining reasonable starting approximations for some of the $g(u)$ roots.

3. Find all roots of the polynomial $\mathcal{P}(z)$ or $Q(w)$ in the complex plane by Newtonian iterations (this task dominates, taking about $60 \%$ of computing time).

4. Estimate roots uncertainties, and select those roots that correspond to real orbital positions (and lying on real hyperbola branches).

5. Among these roots, select the one providing the minimum distance and perform Newtonian 2D iterations to refine this minimum distance and its position.

Now let us comment each stage of this sequence in our updated algorithm.

\subsection{Determining polynomial coefficients}

The coefficients of $g(u)$ are computed by DFT as if we always dealt with the $\mathcal{E} \mathcal{E}$ case, just replacing the definition of $g$ appropriately. We compute a set of values $g_{k}=g\left(u_{k}\right)$ for equally spaced $u_{k}$, and then apply the DFT to obtain all $c_{k}$. We use an excessive number of $u_{k}$ to also compute a few $c_{k}$ with $|k|>8$. These quantities should be zero in theory, so they can be helpful to estimate numeric errors in $c_{k}$.

This scheme can be replicated literally in the $\mathcal{E H}$ case, when $g(u)$ is a trigonometric polynomial with real coefficients. In the $\mathcal{H E}$ and $\mathcal{H} \mathcal{H}$ cases $g(u)$ becomes a hyperbolic polynomial, and $u$ attains the meaning of the hyperbolic anomaly. In this case we could compute the coefficients of $\mathcal{P}(z)$ or $Q(w)$ by performing their polynomial interpolation inside a real segment. This would allow us to avoid complex numbers, as all quantities become real-valued. However, this infers dealing with large differences (large $z$ and small $z$ present simultaneously), so we suspected that this scheme might appear less numerically stable. Therefore, we decided to consider $g(u)$ as a trigonometric polynomial of the imaginary argument (which has the meaning of eccentric anomaly). In this case $g_{k}$ become complex quantities, but the DFT should always produce real $c_{k}$, thanks to the property $g(i u)=g^{*}(-i u)$. The latter property can also be used to halve the number of complex multiplications needed for the DFT. The numeric complexity of this DFT remains the same as in the $\mathcal{E} \mathcal{E}$ case, where we used only real multiplications at this stage.

\subsection{Determining starting approximations for the roots}

As shown in (Baluev and Mikryukov, 2019), smart selection of starting approximations to the roots of $g(u)$ may significantly improve performance as well as numeric stability of the algorithm. In this version of the library, we adopt generally the same scheme here, though augmented in several aspects. This general scheme involves approximations for 4 complex roots extracted first, then 4 guaranteed real roots, and for the remaining roots we adopt the same scheme as for the first 4 ones. This allowed us to reduce the cumulative number of Newtonian iterations, though we need to emphasize that this is merely a statistical effect, appearing when a lot of orbit pairs are processed. Some individual orbit pairs may reveal a specific behavior due to peculiar properties of the roots. 
In the $\mathcal{E} \mathcal{E}$ case, we started iterating the first (complex) root from $z_{1}=0$. The second root starts from $z_{2}=1 / z_{1}^{*}$, third from $z_{3}=1 / z_{2}^{*}$ and so on. Thus we intermittently extract small and large roots, approaching the unit circle. This scheme was based on the empiric observation that complex roots concentrate near $z=0$ and $w=0$. This scheme is preserved in the updated algorithm, and extended literally to the $\mathcal{E} \mathcal{H}$ case. However, in the $\mathcal{H} \mathcal{E}$ and $\mathcal{H} \mathcal{H}$ cases we use more specific starting approximations based on the investigation of statistical distribution of the roots in the complex plane (see Fig. 5 discussed below). In particular, in the $\mathcal{H} \mathcal{H}$ case we start $z_{1}$ from $\exp (0.4 \pi i), z_{2}$ is started from $z_{1}^{*}, z_{3}$ is started from $+i$, $z_{4}$ from $z_{3}^{*}$. In the $\mathcal{H E}$ case the value $+i$ is replaced by $\exp (0.6 \pi i)$.

Starting approximation for the 4 guaranteed real roots are based on the assumption that critical points are often located not far from the orbital nodes. But the hyperbolic orbits introduce additional difficulties in this concern. In particular, we should take care of the real/imaginary hyperbola branches. In the $\mathcal{H} \mathcal{H}$ case exactly one starting point should be placed per each branch combination, because we cannot guarantee that any combination has more than a single critical points. In the $\mathcal{H} \mathcal{E}$ and $\mathcal{E} \mathcal{H}$ case we should distribute two starting points per hyperbolic branch. So, depending on the orbital combination, we adopt the following sets of starting approximations for critical points:

1. $\mathcal{E} \mathcal{E}$ : two internodal distances, each lying entirely on either side from the focus and two distances connecting points separated by $\pm \pi / 2$ from the nodes (in true anomaly), and also lying on the same side from the focus. The first pair usually corresponds to two local minima, while the second one usually describes saddle points.

2. $\mathcal{E} \mathcal{H}$ or $\mathcal{H} \mathcal{E}$ : if each $\mathcal{H}$-branch passes through just a single node then, per each branch, select one internodal distance lying entirely on the same side from the focus (usually minimum), and another internodal distance containing the focus (usually saddle point).

3. $\mathcal{H} \mathcal{H}$ : select all 4 possible internodal distances, if they all refer to different branch combina- tions (that is, if each hyperbolic branch passes through just a single orbital node).

4. The conditions above may be violated if one or both hyperbolic orbits pass through both its nodes simultaneously, while the corresponding imaginary branch does not intersect the other orbital plane at all. In this case half of starting approximations should necessarily involve this imaginary branch. Its orbital position is selected relatively arbitrarily, through a halfsum of hyperbolic anomalies corresponding to the two nodes on the real branch.

Approximations of this "internodal type" should be good for highly inclined orbit pairs, and they revealed good efficiency in the testcase from (Baluev and Mikryukov, 2019). However, it appeared that we did not properly transform angular elements to radians in that testcase (see Corrigendum to Baluev and Mikryukov 2019). This does not mean that initial test results were wrong, as they just corresponded to different testcase. But in the corrected one such initial approximations appeared less efficient and did not reduce the number of Newtonian iterations as desired. Obviously, this appeared because of typically small orbital inclination in the asteroid belt. Also, it appeared that such starting solution are less efficient for hyperbolic orbits as well. We solved the issue by pre-refining every starting approximation through a single 2D Newtonian iteration of $\rho\left(u, u^{\prime}\right)$.

One might argue that this is no better than to make more Newtonian iterations on $\mathcal{P}(z)$, but in actuality $\mathcal{P}(z)$ is less efficient to iterate, because it typically contains close roots. Such an effect appears because each nodal $u$ can be paired with either of two nodal $u^{\prime}$. That is, internodal critical points of $\rho\left(u, u^{\prime}\right)$ usually project to two close pairs when considered in terms of just $u$ or just $u^{\prime}$. But when dealing with $\rho\left(u, u^{\prime}\right)$ they are separated well, thus avoiding the effect of close roots. Hence, even a single 2D iteration allowed to determine a good starting approximation for $u$. In turn, this approach allowed us to save $\sim 20-30$ Newtonian iteration of $\mathcal{P}(z)$ by the cost of making just 4 additional 2D iterations of $\rho\left(u, u^{\prime}\right)$.

The rest of the roots is determined automatically: starting from near-zero $z$ or near-zero $w$ in case of complex $c_{k}(\mathcal{E} \mathcal{E}$ or $\mathcal{E} \mathcal{H})$, or from a random point on 
unit circle for real $c_{k}(\mathcal{H} \mathcal{E}$ or $\mathcal{H} \mathcal{H})$. The latter choice was motivated by the observation that real roots are often more difficult to locate without a good starting approximations.

\subsection{Finding all complex roots}

The roots of $\mathcal{P}(z)$ are found one by one, by means of running the Newtonian root search with subsequent division of $\mathcal{P}(z)$ by the corresponding linear factor $z-z_{k}$. we introduced a few minor changes to this scheme.

First, we extract the roots until a quartic polynomial is obtained, rather then a quadratic one. The last four roots of the quartic polynomial are computed using explicit formulae of the Ferrari method. Since these formulae are more complicated and may have a reduced numeric stability sometimes, we also refine each of these 4 roots by a single Newtonian iteration applied to that quartic polynomial. Solving the quartic polynomial in such a way appeared a bit faster.

Second, to further improve numeric stability, we apply Newton iterations to either $\mathcal{P}(z)$, if $|z|<1$, or $Q(w)$, if $|z|>1$. In the previous version of the algorithm we rejected this approach because it frequently lead to looping conditions, when we infinitely jump between the same points. Now we avoided this issue by performing the mode change in a more "lazy" manner. Namely, we keep the $|z|<1$ mode until $|z|^{2}$ reaches 5 , and the mode $|z|>1$ is kept while $|z|^{2}>1 / 2$.

Third, while in the elliptic case the paired complex roots obey the rule $z_{2}=1 / z_{1}^{*}=z_{1} /\left|z_{1}\right|^{2}$, in case when the first orbit is hyperbolic it should be replaced by $z_{2}=z_{1}^{*}$.

\subsection{Real roots selection}

In the $\mathcal{E} \mathcal{E}$ and $\mathcal{E} \mathcal{H}$ cases $g(u)$ is a trigonometric polynomial, so we should select the roots lying on the unit circle $|z|=|w|=1$. We may then use the same criterion as in (Baluev and Mikryukov, 2019):

$$
\Delta_{z}=\frac{|\log | z||}{v \varepsilon_{z}} \leq 3,
$$

where $v$ is a manual normalizing factor and $\varepsilon_{z}$ is the relative root uncertainty (estimated literally as in
Baluev and Mikryukov 2019). This criterion can be rewritten as

$$
|\mathfrak{R} \log z| \leq 3 v \varepsilon_{z} .
$$

Now we have $\mathcal{H E}$ and $\mathcal{H} \mathcal{H}$ cases, in which $g(u)$ is a hyperbolic polynomial. The coefficients of $\mathcal{P}(z)$ are real, and we are interested in only real positive $z$ (that correspond to real $u$ ). In this case the criterion becomes

$$
|\mathfrak{J} \log z| \leq 3 v \varepsilon_{z}
$$

so in place of (6) we have

$$
\Delta_{z}=\frac{|\arg z|}{v \varepsilon_{z}} \leq 3
$$

Notice that this criterion also filters away all roots corresponding to the imaginary branch of $\mathcal{H}$ (negative $z$ ). Still, in $\mathcal{H} \mathcal{H}$ case it might occur that some roots with $z>0$ correspond to the imaginary branch of the second orbit $\mathcal{H}^{\prime}$. We may then obtain negative $\cosh u^{\prime}$.

\subsection{Final MOID refining and testing the reliability}

Each selected root $z_{k}$ implies the orbital position as $u_{k}=\arg z_{k}$, or $u_{k}=-\log z_{k}$. The orbital position on $O^{\prime}$ is determined using formulae from (Baluyev and Kholshevnikov, 2005). After that, the both orbital positions may still involve increased numeric errors and should be refined using 2D Newtonian iterations. The scheme here remains entirely the same as in (Baluev and Mikryukov, 2019), with obvious replacements for the hyperbolic motion. The formulae determining numeric uncertainty of the result are also nearly the same, with the only difference that $\rho\left(u, u^{\prime}\right)$ and the product $a a^{\prime}$ are negative in the mixed $\mathcal{E} \mathcal{H} / \mathcal{H} \mathcal{E}$ cases. They all should be replaced by absolute values in that formulae.

Finally, we apply the following sequence of checks to verify the reliability of the results:

1. All roots that passed (6) or (9) must comply with the requested least accuracy: $v \varepsilon_{z}<\delta_{\max }$.

2. The minimum of $\Delta_{z}$ among all the roots that failed (6) or (9) must exceed 10, meaning that there is no other suspicious root candidates. That is, the families of selected real and other roots must be separated by a clear gap. 
3. The number of roots that passed (6) or (9) must be even and no smaller than 4 (for $\mathcal{E} \mathcal{E}$ and $\mathcal{E H}$ cases), or even and no smaller than 2 (for $\mathcal{H} \mathcal{E}$ and $\mathcal{H} \mathcal{H}$ cases).

4. After the $2 \mathrm{D}$ refining, the Hessian $\mathbf{H}_{\mathrm{rsd}}$ of $\rho\left(u, u^{\prime}\right)$ is positive-definite (for $\mathcal{E} \mathcal{E}$ and $\mathcal{H} \mathcal{H}$ cases), or negative-definite (for $\mathcal{E} \mathcal{H}$ and $\mathcal{H} \mathcal{E}$ cases).

5. On the $2 \mathrm{D}$ refining stage, the total cumulative change in $u$ satisfies the condition $|\Delta u|<\delta_{\max }$.

They mostly replicate those from (Baluev and Mikryukov, 2019), with minor corrections.

\subsection{Extending the fallback algorithm to hyperbolas}

In addition to the basic fast method based on $g(u)$ root-finding, the DISTLINK library implemented a brute force-like minimization of the distance function $\rho\left(u, u^{\prime}\right)$ with respect to $u$ (the other position $u^{\prime}$ is determined through $u$ analytically). This more slow method can be used as an additional alternative when the basic one signals a warning. In this work we generalized this method to the hyperbolic orbits as well. The general scheme remains entirely the same as in (Baluev and Mikryukov, 2019). The changes are only due to the scan range for $u$ which is determined by new formulae. Its method was based on an observation that MOID is usually located close to the orbital nodes, and this idea is further developed in Appendix A for orbit pairs involving hyperbolas. As in the elliptic case, the algorithm may automatically swap the orbits to scan a smaller range in $u$.

\section{Practical validation and benchmarks}

We tested our algorithm on the first 10000 numbered asteroids from the Lowell observatory catalogue astorb. dat ${ }^{1}$. This implies $\sim 10^{8}$ orbit pairs. We used exactly the same version of the catalogue as in (Baluev and Mikryukov, 2019), but here we correctly transform angular elements (see Corrigendum to that work).

To obtain hyperbolic orbits, we transformed the asteroid orbital eccentricities as $e \mapsto 1-\log e$, changing the sign of semimajor axis. In this way we

\footnotetext{
${ }^{1}$ See url ftp://ftp.lowell.edu/pub/elgb/astorb.html
}

Table 1: Frequency of potentially unreliable occurrences.

\begin{tabular}{|c|c|c|}
\hline case & single $\operatorname{MOID}\left(O, O^{\prime}\right)$ & $\begin{array}{l}\operatorname{MOID}\left(O, O^{\prime}\right) \text { and } \\
\operatorname{MOID}\left(O^{\prime}, O\right)\end{array}$ \\
\hline $\mathcal{E} \mathcal{E}$ & one per 14600 & one per 413000 \\
\hline $\mathcal{E H}$ & one per 66000 & one per $10^{8}$ \\
\hline $\mathcal{H E}$ & one per 348000 & - as above - \\
\hline $\mathcal{H} \mathcal{H}$ & one per 2580 & one per 55900 \\
\hline
\end{tabular}

generated $\sim 10^{8}$ orbit pairs for each of our orbital configurations: $\mathcal{E} \mathcal{E}, \mathcal{E} \mathcal{H}, \mathcal{H} \mathcal{E}$, and $\mathcal{H} \mathcal{H}$. Notice that computation is asymmetric in its nature both for our algorithm and for the Gronchi (2005) code, so the result may appear different for $\operatorname{MOID}\left(O, O^{\prime}\right)$ for $\operatorname{MOID}\left(O^{\prime}, O\right)$. Normally, this should be a small difference due to only numeric errors, but it may appear large if we faced some serious algorithm failure like missing critical point.

In this work we used the Intel Core i7 configuration explained in (Baluev and Mikryukov, 2019) and the standard DoubLE floating-point arithmetic (unless otherwise stated).

The frequency of unreliable cases that failed some of the post-computing requirements above, are given in Table 1. Notice that these frequencies in the $\mathcal{E} \mathcal{E}$ case appeared somewhat higher than mentioned in (Baluev and Mikryukov, 2019) because the new values refer to the corrected test case which has more near-coplanar occurrences. The number of potentially unreliable computations is increased in the $\mathcal{H} \mathcal{H}$ case because it frequently infers that MOID segment is located rather far from the focus, basically between the asymptotes as skew lines. In the degenerated case when some asymptotes are parallel, the MOID can be even achieved at the infinity (Baluyev and Kholshevnikov, 2005). In such cases, additional numeric difficulties appear because of subtraction of large close vectors when computing the MOID. This results in additional numeric errors causing the violation of our post-condition that error in $u$ should be below the maximum allowed error. This effect seems difficult to avoid, but from the other side such violation appears rather formal, because it is unrelated to possible lost roots.

Among occurrences that were reported as unreliable in either $\operatorname{MOID}\left(O, O^{\prime}\right)$ or $\operatorname{MOID}\left(O^{\prime}, O\right)$ run, nearly all appeared to have correct MOID in turn. 
In the $\mathcal{E} \mathcal{E}$ run we detected only 11 occurrences when either of the two runs gave wrong distance. However, in all that cases the larger distance was always flagged properly as unreliable, while the other one appeared in agreement with the MOID provided by Gronchi (2005) code. In all the cases when both computations were flagged as unreliable, both MOIDs actually appeared correct. There were only 5 occurrences with wrong distance in the $\mathcal{H E} / \mathcal{E H}$ case, and 4 ones in the $\mathcal{H} \mathcal{H}$ one. All such events were properly flagged as well.

We provide a comparison of our algorithm with the Gronchi code in Fig. 1. We compute the differences of the Gronchi code MOID minus the MOID obtained by our code. If either algorithm self-diagnosed a warning/failure, we removed this occurence from the consideration. We may see that in every orbital combination there are multiple occurrences when Gronchi code obtained clearly overestimated MOID value (i.e., it missed the true global minimum). We did not detect opposite occurrences, possibly except for those with difference below $10^{-11}$ AU (they are simply the effect of roundoff errors). Summarizing, we did not find an occurence in which the DISTLINK would yield clearly wrong MOID value without setting the unreliability flag.

We also considered another test setting when we compute the MOID twice by swapping the orbits. The DISTLINK algorithm may be called second time only if the first computation signaled a warning, so this does not essentially slow down the cumulative performance. The Gronchi code, however, does not seem to diagnose its failures well, as we have demonstrated above. Therefore, we always run it twice in this test, roughly doubling the computing time. Still, there are orbit pairs that could not be processed well by Gronchi code even with such swapping, while our code yielded significantly smaller MOID on them (see Fig. 2). We found such pairs only in the mixed $\mathcal{E} \mathcal{H} / \mathcal{H} \mathcal{E}$ case, while the $\mathcal{E} \mathcal{E}$ and $\mathcal{H} \mathcal{H}$ cases demonstrated good agreement between DISTLINK and Gronchi code. However, such pairs were found the initial (uncorrected) testcase from (Baluev and Mikryukov, 2019) in the $\mathcal{E} \mathcal{E}$ case.

In Fig. 3 we compare the quadrature sum of the reported MOID uncertainties,
$\sigma_{\mathrm{MOID}}=\sqrt{\sigma_{\mathrm{MOID}\left(\mathcal{E}, \mathcal{E}^{\prime}\right)}^{2}+\sigma_{\mathrm{MOID}\left(\mathcal{E}^{\prime}, \mathcal{E}\right)}^{2}}$, with the difference $\left|\operatorname{MOID}\left(\mathcal{E}, \mathcal{E}^{\prime}\right)-\operatorname{MOID}\left(\mathcal{E}^{\prime}, \mathcal{E}\right)\right|$ that can be deemed as an empiric estimate of the actual MOID error. We may conclude that our algorithm provides rather safe and realistic assessment of numeric errors, intentionally somewhat pessimistic. We found just two occurrences when the empiric error slightly exceeded the predicted uncertainty, but this violation appears very formal and insignificant. Notice that initially we did not expect to put a strict error limit, this behaviour appears partly curious.

In Fig. 4 we provide additional statistical distributions related to our algorithm: the number of Newtonian iterations of $\mathcal{P}(z)$, of 2D Newtonian iterations, and of the total number of real roots of $g(u)$.

Finally, we investigated the distributions of the roots in the complex plane (in terms of $z$ variable). They are plotted in Fig. 5 in the form of 2D histograms, for all 4 orbital combinations. We can see that whenever $g(u)$ is trigonometric $(\mathcal{E} \mathcal{E}$ and $\mathcal{E} \mathcal{H})$, complex roots concentrate near $z=0$ (and, concequently, near $w=0$ or $z=\infty$ ). The real (in terms of $u$ ) roots are well separated from the complex ones, there is rather small density near the unit circle (except for this circle itself). As expected, these two distributions are nearly radially symmetric, the roots do not reveal remarkable concentrate in any angular directions.

The cases with hyperbolic $g(u)$ appear more intriguing. In this case real (in terms of $u$ ) roots lie on the real positive ray $z>0$, but we can see quite complicated and asymmetric structure in the complex domain, especially detailed near the unit circle. In the $\mathcal{H} \mathcal{H}$ case we can see 4 obvious concentrations: two at $z= \pm i$ and two with $|z|=1$ and $\arg z \approx \pm 0.4 \pi$, while $z= \pm 1$ are avoided (except for purely real roots). In the $\mathcal{H} \mathcal{E}$ case, the $z= \pm i$ concentrations are replaced by those with $|z|=1$ and $\arg z \approx \pm 0.6 \pi$. It is unclear, how much this puzzling structure is sensitive to our particular testcase. The primary narrow concentrations are difficult to explain by statistical properties of test orbits.

In Table 2, we present our performance benchmarks for this test application. Here we used either 80-bit LONG DOUBLE floating-point arithmetic or the 64-bit DOuBLE one, with all details the same as 

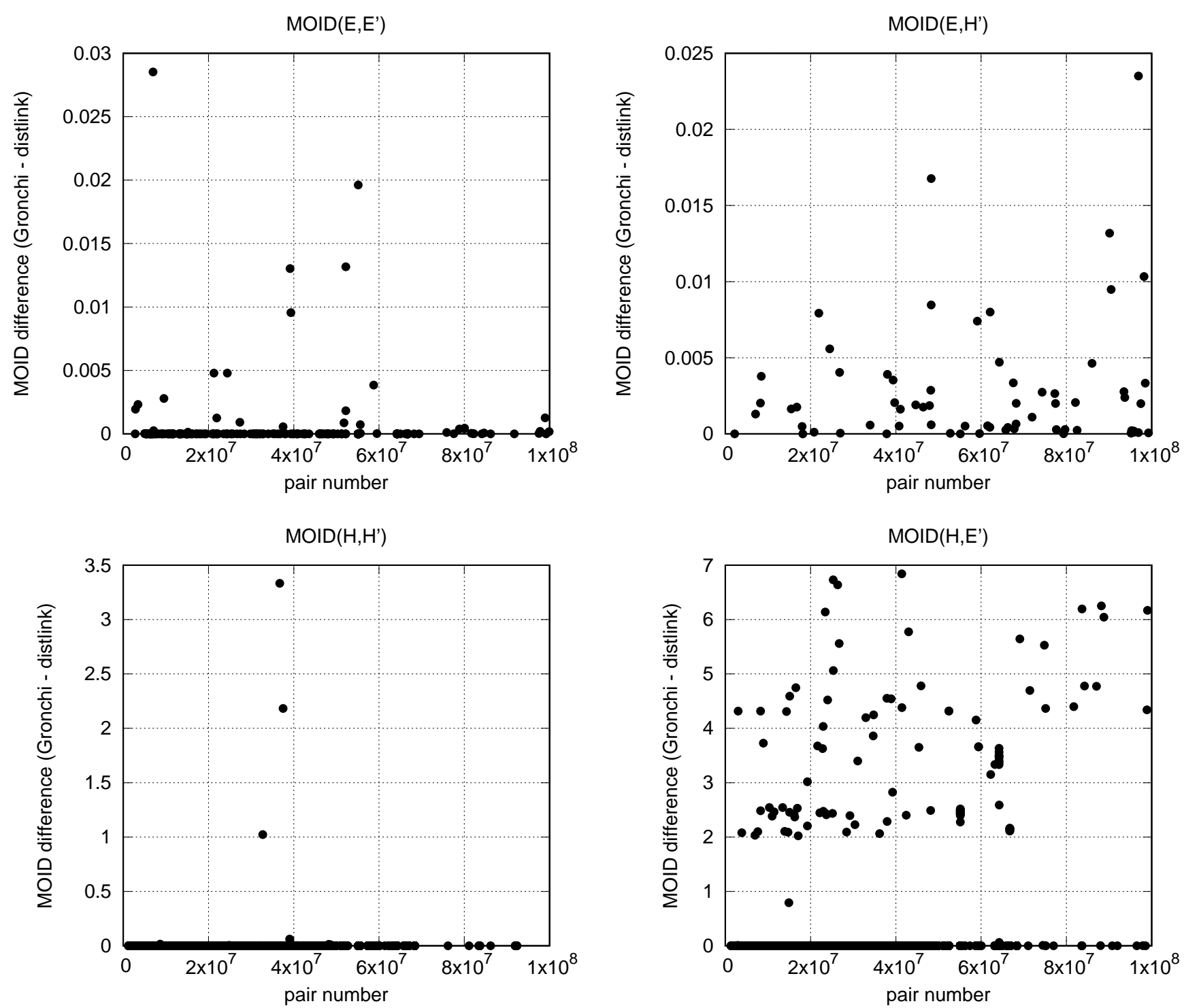

Figure 1: The difference between MOID values computed by the Gronchi's code and by our algorithm (labelled as DISTLINK). Panels refer to different orbital combinations as labelled. All differences smaller than $10^{-11}$ AU (in absolute value) were removed. No points below zero were detected in these conditions, meaning that our code always returned smaller value than the Gronchi's one.

in (Baluev and Mikryukov, 2019). The code was compiled with GCC and optimized for the local CPU architecture using the same flags as well (-03 -march=native -mf pmath=sse). However, the OS was upgraded since that time, including the GCC and GLIBC packages. This might cause a subtle increase of the performance, $1 \mu \mathrm{s}$ for the Gronchi code (and possibly similar for the DISTLINK).

We conclude that our algorithm remains quite competitive in terms of speed. It outperformes the Gronchi code, though rather marginally if the first orbit is hyperbolic. For the both algorithms, the $\mathcal{H E}$ configurations appears more slow than $\mathcal{E H}$, so the latter one is preferred in practice. The exact reason why there is a remarkable performance drop in our code on a hyperbolic first orbit is still unclear, perhaps there is still some room to increase the speed in such configurations. The minor algorithm improvements listed in Sect. 3 had only a negligible effect in case of the Double arithmetic, but their effect is more remarkable in the LONG DOUBLE framework, improving the performance by $10-15 \%$. 
Table 2: Performance tests on the first 10000 asteroids from the Main Belt: average CPU time per MOID.

\begin{tabular}{lcccc}
\hline case & \multicolumn{2}{c}{ DOUBLE arithmetic } & \multicolumn{2}{c}{ LONG DOUBLE arithmetic } \\
& $\begin{array}{c}\text { DISTLINK } \\
\text { (fast alg.) }\end{array}$ & Gronchi code & $\begin{array}{c}\text { DISTLINK } \\
\text { (fast alg.) }\end{array}$ & Gronchi code \\
\hline $\mathcal{E}$ & $23 \mu \mathrm{s}$ & $33 \mu \mathrm{s}$ & $69 \mu \mathrm{s}$ & NA \\
$\mathcal{E H}$ & $22 \mu \mathrm{s}$ & $28 \mu \mathrm{s}$ & $76 \mu \mathrm{s}$ & NA \\
$\mathcal{H} \mathcal{E}$ & $30 \mu \mathrm{s}$ & $32 \mu \mathrm{s}$ & $84 \mu \mathrm{s}$ & NA \\
$\mathcal{H} \mathcal{H}$ & $29 \mu \mathrm{s}$ & $31 \mu \mathrm{s}$ & $76 \mu \mathrm{s}$ & NA \\
\hline
\end{tabular}

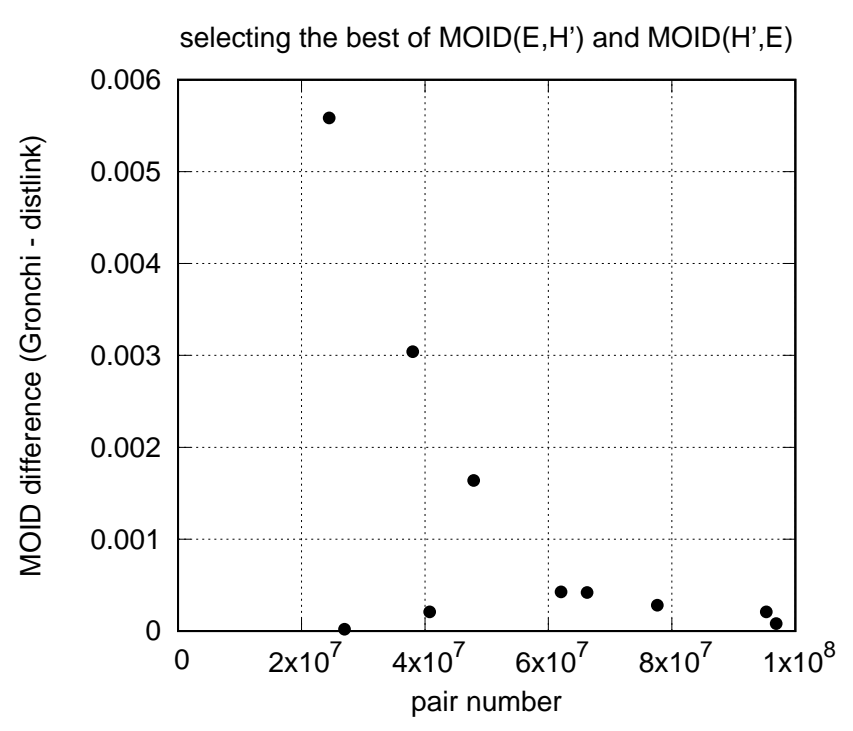

Figure 2: The difference between MOID values computed by the Gronchi's code and by our algorithm (labelled as DISTLINK). Similar to Fig. 1, but here we compute the MOID twice by swapping the orbits and select the "best" result (see text). Only the mixed $\mathcal{H} \mathcal{E} / \mathcal{E} \mathcal{H}$ case is shown, because there were none significant differences in the other two combinations.

\section{Conclusions}

In the end we would like to discuss further paths of developing the DISTLINK algorithm. We did not yet consider parabolic orbits and other degenerate cases. However, there are many comets moving on nearparabolic, and even if their eccentricity is not precisely unit, it can be approximated by unit at some early stage. From the analytic theory, if either $e=1$ or $e^{\prime}=1$ the algebraic degree of the associated polynomial is reduced to 12 , and whenever both the orbits are parabolic the algebraic degree becomes 9 (Baluyev and Kholshevnikov, 2005). This would imply certain simplifications and likely significant speedup of the computation. However, this also requires a more specific treatment of such cases due to the degeneracy.

Some performance increase to the basic algorithm is still possible. It spends significant time on finding complex roots of $g(u)$, and therefore one may try to eliminate this unnecessary work at least partly. This is unfortunately not so easy because we cannot control the order in which the roots are extracted. Though we made some efforts to extract 4 guaranteed real roots in the beginning, this optimization is of a statistical type, i.e. it works in average but frequently fails for individual configurations. However, to increase the average performance one may simply stop extracting the roots if the polynomial has none real roots.

In case when $g(u)$ is hyperbolic with real coefficients $c_{k}$, this can be verified by the Descartes' rule of signs, that is, if the coefficients $c_{k}$ are all positive or all negative then there are no positive real roots. In case of trigonometric $g(u)$, when $c_{k}$ are complex, one need to verify the existence of roots with $|z|=1$. This can be done based on the available classic upper and lower bounds on the polynomial roots; for example, if at some point $\left|z_{k}\right|<z_{\max }$ and $z_{\max }<1$ then there is no roots with $|z|=1$, hence no real roots remain in $g(u)$. However, approaches of this type require to track uncertainties in each $c_{k}$ which are accumulated after every root extraction (division of $\mathcal{P}(z)$ by $z-z_{k}$ ). This may result in a loss of numeric reliability due to unavoidable assumptions about errors, so implementing this method would require a detailed investigation of possible side effects.

Another way to increase the performance is optimization of complex-valued arithmetics. It is cur- 

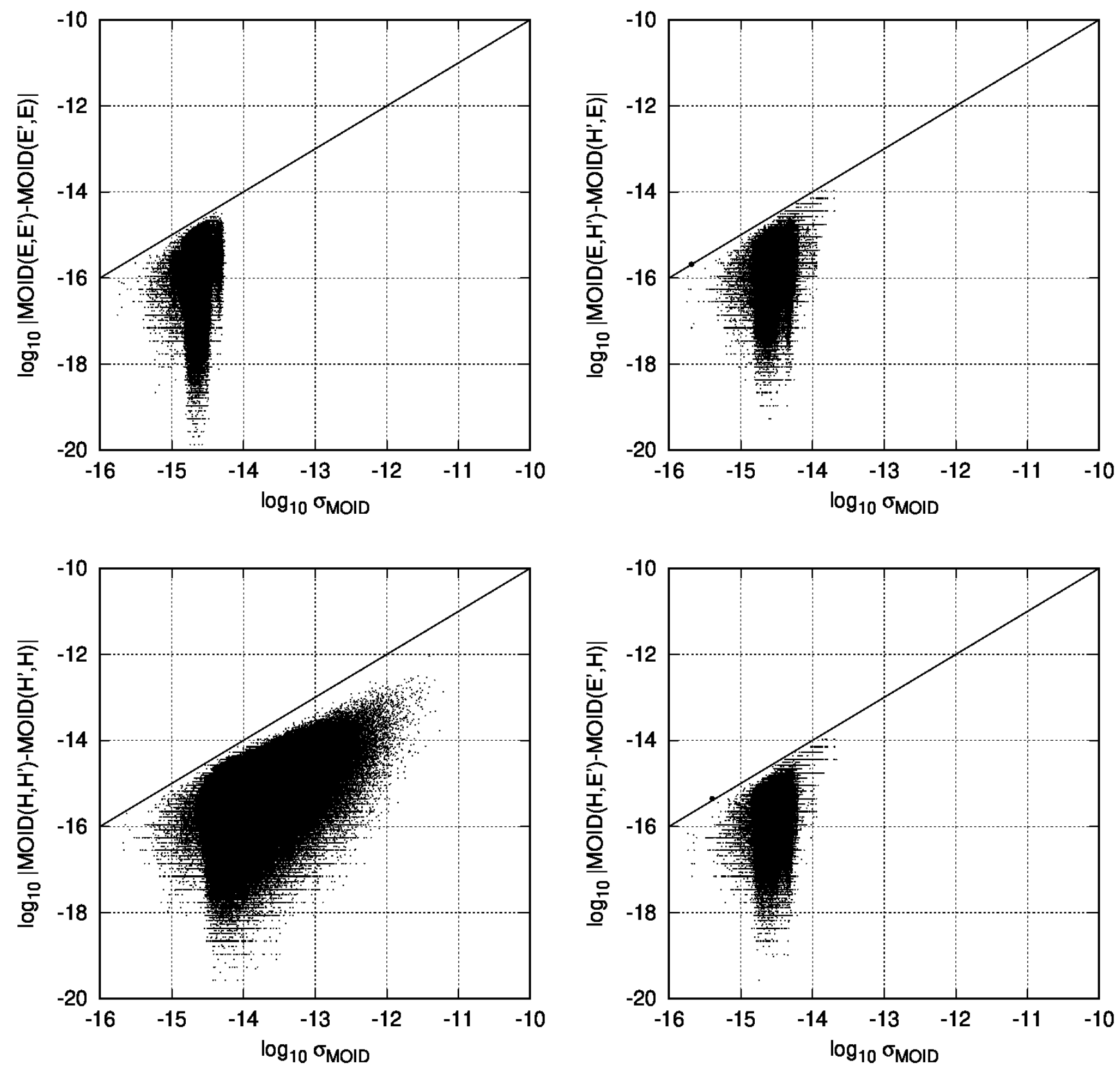

Figure 3: Distribution of the estimated uncertainties $\sigma_{\text {MOID }}$ versus an empiric error measure $\left|\operatorname{MOID}\left(O, O^{\prime}\right)-\operatorname{MOID}\left(O^{\prime}, O\right)\right|$. Panels refer to different orbital combinations as labelled $(\mathcal{H} \mathcal{E}$ and $\mathcal{E} \mathcal{H}$ cases should be statistically equivalent here).

rently based on the build-in $\mathrm{C}++$ library (through class complex). This implementation appears numerically reliable (at least for GCC), however it includes significant overheads due to additional checks related to correct support of $\mathrm{NaN} / \mathrm{Inf}$ arithmetic and over/underflows. We noticed a roughly double speedup whenever these extra checks are turned off (the GCC flag-ffast-math). The complex multiplication is then reduced to the school grammar for- mula $z w=(\mathfrak{R} z \mathfrak{R} w-\mathfrak{J} z \mathfrak{J} w)+i(\mathfrak{R} z \mathfrak{J} w+\mathfrak{J} z \mathfrak{R} w)$. In this way our library unfortunately generated frequent wrong results without warnings, indicating its sensitivity to correct handling of such subtle arithmetic issues. Nevertheless, a remarkable performance increase can be achieved if the causes of such sensitivity are located in the code and eliminated. 

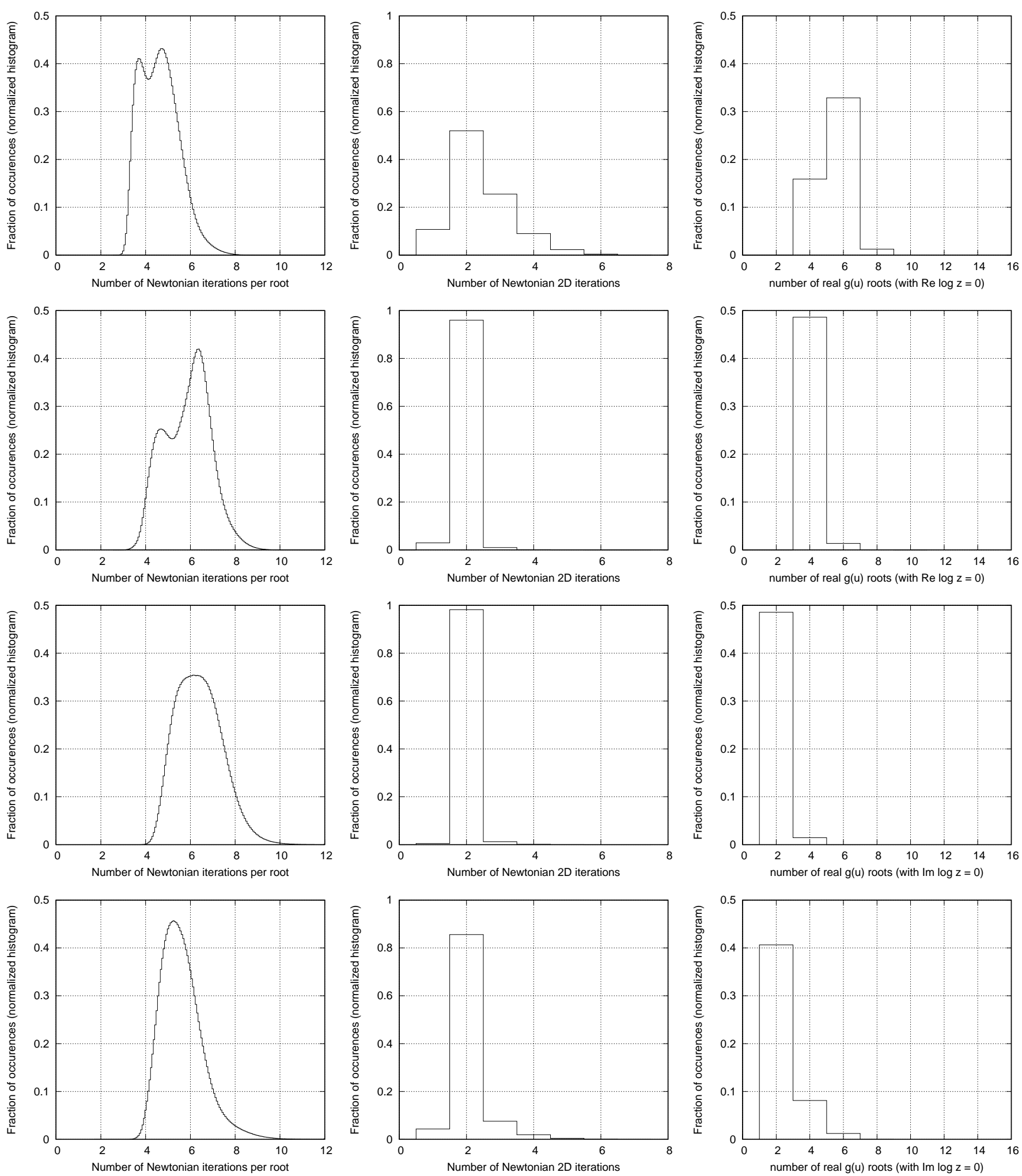

Figure 4: Histograms for the number of Newtonian iterations spent per root of $g(u)$ (left), of 2D Newtonian iterations on the refine stage (middle), and for the number of real roots. The four rows refer to the $\mathcal{E}, \mathcal{E} \mathcal{H}, \mathcal{H} \mathcal{E}, \mathcal{H} \mathcal{H}$ cases (top to bottom). The histograms were normalized by the bin width to render the probability density function for the quantity labelled in the abscissa.

\section{Acknowledgements}

press gratitude to Dr. G.F. Gronchi for reviewing this

This work was supported by the Russian Sci-

ence Foundation grant no. 18-12-00050. We ex-12 

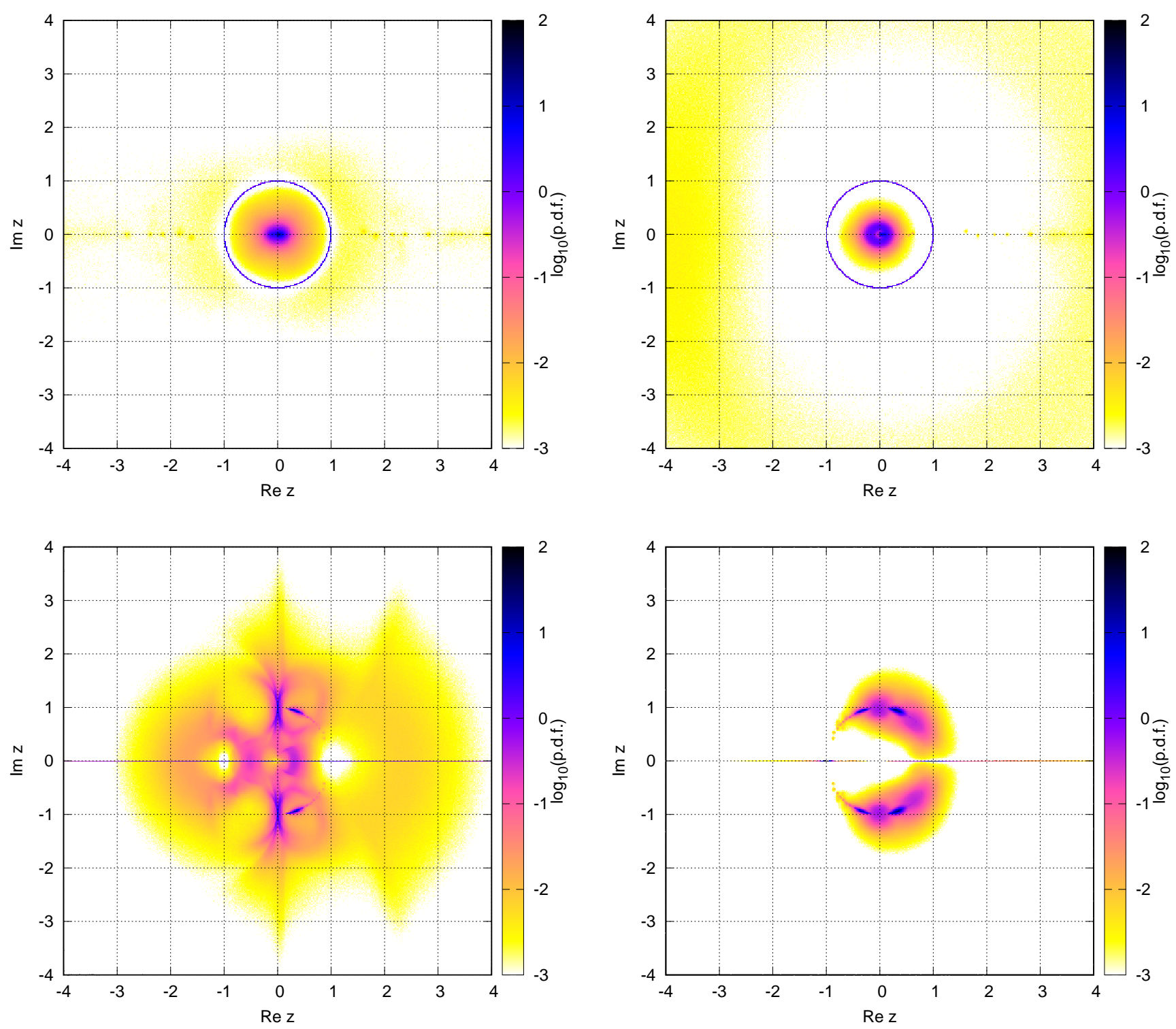

Figure 5: Distribution of $g(u)$ roots in the complex $z$-plane, based on our primary testcase. Panels show the $\mathcal{E} \mathcal{E}$ (top-left), the $\mathcal{E} \mathcal{H}$ (top-right), the $\mathcal{H} \mathcal{E}$ (bottom-right), and the $\mathcal{H} \mathcal{H}$ (bottom-left) cases. The color reflects the level of normalized 2D histogram that estimates the corresponding probability density function (p.d.f.)

manuscript and providing useful comments.

\section{Appendix A. Determining the scan range on a hyperbolic orbit}

Let us introduce vector $\boldsymbol{W}$, which is directed to the ascending node of $O^{\prime}$ assuming reference $O$, and has length $W=\sin I$, where $I$ is mutual inclination between the orbits. The components of $\mathbf{W}$ are given in (Baluev and Mikryukov, 2019). Then the true anomaly of this node in $O$ is $\theta_{\Omega}$, where

$$
\cos \theta_{\Omega}=(P W) / W, \quad \sin \theta_{\Omega}=(Q W) / W,
$$

while $\boldsymbol{P}$ and $\boldsymbol{Q}$ are orthogonal unit vectors in the orbital plane (Baluyev and Kholshevnikov, 2005, Baluev and Mikryukov, 2019). The location on the other orbit $\theta_{\Omega}^{\prime}$ can be determined in a similar way through $\boldsymbol{P}^{\prime}$ and $\boldsymbol{Q}^{\prime}$. 
After that let us compute

$$
\begin{aligned}
r_{ \pm} & =\frac{p}{1 \pm e \cos \theta_{\Omega}}, & r_{ \pm}^{\prime} & =\frac{p^{\prime}}{1 \pm e^{\prime} \cos \theta_{\Omega}^{\prime}}, \\
d_{1} & =r_{+}-r_{+}^{\prime}, & d_{2} & =r_{-}-r_{-}^{\prime}, \\
d_{3} & =r_{+}+r_{-}^{\prime}, & d_{4} & =r_{-}+r_{+}^{\prime}, \\
d_{\Omega} & =\min _{r_{ \pm}, r_{ \pm}^{\prime}>0}\left(\left|d_{1}\right|,\left|d_{2}\right|,\left|d_{3}\right|,\left|d_{4}\right|\right), & &
\end{aligned}
$$

where the minimum is considered only among $d_{k}$ that do not involve negative radii. Notice that $\theta_{\Omega}$ or $\theta_{\Omega}^{\prime}$ may lie on the imaginary hyperbola branch with negative $r_{+}$or $r_{+}^{\prime}$, but in such a case $r_{-}$or $r_{-}^{\prime}$ would be necessarily positive, and vice versa. It is also possible that both $r_{ \pm}$(or $r_{ \pm}^{\prime}$ ) appear positive simultaneously. In (Baluev and Mikryukov, 2019) we used only the internodal distances $d_{1}$ and $d_{2}$ to compute $d_{\Omega}$, but $d_{3}$ and $d_{4}$ may appear smaller in some cases (Mikryukov and Baluev, 2019). In the hyperbolic case it is important to take into account $d_{3,4}$, because it may appear that $d_{1,2}$ both involve imaginary branches.

If $O$ is an ellipse, the rest remains the same as in (Baluev and Mikryukov, 2019). Let us define the quantity $k \geq 0$ and the angle $\varphi$ from

$$
\begin{array}{r}
A^{2}=1-e^{2} \cos ^{2} \theta_{\Omega}, \quad k=\frac{d_{\Omega}}{a W A}, \\
\sin \varphi=\frac{\sin \theta_{\Omega}}{A}, \quad \cos \varphi=\sqrt{1-e^{2}} \frac{\cos \theta_{\Omega}}{A},
\end{array}
$$

then the necessary $u$ range is derived from the inequality

$$
e \sin \varphi-k \leq \sin (\varphi-u) \leq e \sin \varphi+k .
$$

It has three types of solutions discussed in (Baluev and Mikryukov, 2019). The only possible difference here is due to a refined $d_{\Omega}$ in (A.2).

Whenever $O$ is a hyperbola, the matters get more complicated. Then we have

$$
\begin{array}{r}
e m-\frac{d_{\Omega}}{|a| W} \leq m \cosh u+n \sinh u \leq e m+\frac{d_{\Omega}}{|a| W}, \\
m=\sin \theta_{\Omega}, \quad n=\cos \theta_{\Omega} \sqrt{e^{2}-1},
\end{array}
$$

and the solution depends on the relationship between $|m|$ and $|n|$.

$$
\begin{aligned}
& \text { If }\left|\tan \theta_{\Omega}\right|>\sqrt{e^{2}-1}, \text { we put } \\
& \qquad \begin{array}{r}
A^{2}=1-e^{2} \cos ^{2} \theta_{\Omega}, \quad k=\frac{d_{\Omega}}{|a| W A}, \\
\tanh \varphi=\sqrt{e^{2}-1} \cot \theta_{\Omega},
\end{array}
\end{aligned}
$$

Then the inequality becomes

$$
e \cosh \varphi-k \leq \cosh (\varphi+u) \leq e \cosh \varphi+k .
$$

This inequality determines two types of ranges for $u$ :

1. If $e \cosh \varphi>1+k$ then there are two segments surrounding the nodes, $[\operatorname{arcosh}(e \cosh \varphi-$ $k)-\varphi, \operatorname{arcosh}(e \cosh \varphi+k)-\varphi]$ and $[-\operatorname{arcosh}(e \cosh \varphi+k)-\varphi,-\operatorname{arcosh}(e \cosh \varphi-$ $k)-\varphi]$.

2. If $e \cosh \varphi \leq 1+k$ then there is a $\sin$ gle large segment $[-\operatorname{arcosh}(e \cosh \varphi+k)-$ $\varphi, \operatorname{arcosh}(e \cosh \varphi+k)-\varphi]$.

Otherwise, if $\left|\tan \theta_{\Omega}\right|<\sqrt{e^{2}-1}$, we define

$$
\begin{array}{r}
A^{2}=e^{2} \cos ^{2} \theta_{\Omega}-1, \quad k=\frac{d_{\Omega}}{|a| W A}, \\
\operatorname{coth} \varphi=\sqrt{e^{2}-1} \cot \theta_{\Omega},
\end{array}
$$

and the inequality is

$$
e \sinh \varphi-k \leq \sinh (\varphi+u) \leq e \sinh \varphi+k .
$$

This always defines a single segment $u \in$ $[\operatorname{arsinh}(e \sinh \varphi-k)-\varphi, \operatorname{arsinh}(e \sinh \varphi+k)-\varphi]$.

Finally, let us consider the degenerate case $\left|\tan \theta_{\Omega}\right|=\sqrt{e^{2}-1}$, inferring that one of the nodes coincides with an asymptote of $O$. This is a degenerate case, so we presently do not include it in our code, but still it might be interesting for the sake of completeness. In this case one of $r_{ \pm}$is infinite, but the other one $r_{\mp}=p / 2$ is then finite and positive, so $d_{\Omega}$ should remain finite. It remains finite even if this degeneracy is present on the both orbits simultaneously (and hence, orbits have a common asymptote). We have now $A=0$ and $\varphi=\infty$, so we put $k=\left(e d_{\Omega}\right) /\left(|a| W \sqrt{e^{2}-1}\right)$, and then

$$
e-k \leq \exp ( \pm u) \leq e+k
$$

where the sign coincides with the sign of $\tan \theta_{\Omega}$. There are two types of the $u$-range:

1. If $k>e$ then $\pm u \in[\log (e-k), \log (e+k)]$.

2. If $k \leq e$ then $\pm u \leq \log (e+k)$, and this remains to be the only unbounded case. 


\section{References}

Armellin, R., di Lizia, P., Berz, M., Makino, K., 2010. Computing the critical points of the distance function between two Keplerian orbits via rigorous global optimization. Celest. Mech. Dyn. Astron. 107, 377-395.

Baluev, R.V., Mikryukov, D.V., 2019. Fast error-controlling MOID computation for confocal elliptic orbits. Astron.\& Comput. 27, 11-22.

Baluyev, R.V., Kholshevnikov, K.V., 2005. Distance between two arbitrary unperturbed orbits. Celest. Mech. Dyn. Astron. 91, 287-300.

Dybczyński, P.A., Jopek, T.J., Serafin, R.A., 1986. On the minimum distance between two Keplerian orbits with a common focus. Celest. Mech. 38, 345-356.

Gronchi, G.F., 2002. On the stationary points of the squared distance between two ellipses with a common focus. SIAM J. Sci. Comput. 24, 61-80.

Gronchi, G.F., 2005. An algebraic method to compute the critical points of the distance function between two Keplerian orbits. Celest. Mech. Dyn. Astron. 93, 295-329.

Gronchi, G.F., Niederman, L., 2020. On the nodal distance between two Keplerian trajectories with a common focus. Celest. Mech. Dyn. Astron. 132, 5.

Guzik, P., Drahus, M., Rusek, K., Waniak, W., Cannizzaro, G., Pastor-Marazuela, I., 2019. Initial characterization of interstellar comet 2I/Borisov. Nat. Astron. 4, 53-57.

Hedo, J.M., Ruíz, M., Peláez, J., 2018. On the minimum orbital intersection distance computation: a new effective method. MNRAS 479, 3288-3299.

Kholshevnikov, K., Vassiliev, N., 1999. On the distance function between two Keplerian elliptic orbits. Celest. Mech. Dyn. Astron. 75, 75-83.

Mikryukov, D.V., Baluev, R.V., 2019. A lower bound of the distance between two elliptic orbits. Celest. Mech. Dyn. Astron. $131,28$.

Sitarski, G., 1968. Approaches of the parabolic comets to the outer planets. Acta Astronomica 18, 171-195. 